\section{Posição socioeconômica e autoavaliação da saúde bucal no Brasil: resultados da Pesquisa Nacional de Saúde}

\author{
Socioeconomic position and self-rated oral \\ health in Brazil: results of the Brazilian \\ National Health Survey
}

Posición socioeconómica y autoevaluación de

salud bucal en Brasil: resultados de la

Encuesta Nacional de Salud

\section{Resumo}

Analisou-se a autoavaliação da saúde bucal da população adulta brasileira segundo a posição socioeconômica (região de residência, escolaridade, renda e classe social), explorando as variáveis com maior sensibilidade para medir tal associação. Estudaram-se 59.758 indivíduos com 18 anos ou mais de idade, que participaram da Pesquisa Nacional de Saúde 2013, um inquérito domiciliar de base populacional. A autoavaliação da saúde bucal (dentes e gengivas) foi analisada como positiva, regular e negativa. Com base na regressão logística multinomial, estimaram-se odds ratio $(\mathrm{OR})$ brutos e ajustados e os respectivos intervalos de 95\% de confiança (IC95\%). Foram calculados os percentuais de concordância e o valor de kappa para comparar os valores obtidos pelos modelos de regressão e os valores esperados. A prevalência da autoavaliação da saúde bucal positiva foi 67,4\%, 26,7\% para regular e 5,9\% para negativa. Após ajuste, a chance de autoavaliar a saúde bucal como negativa foi significativamente mais elevada entre os indivíduos com renda domiciliar per capita de até um salário mínimo (OR = 4,71; IC95\%: 2,84-7,83), sem nível de escolaridade completo (OR = 3,28; IC95\%: 2,34-4,61), da classe social destituídos de ativos (OR = 3,03; IC95\%: 2,12-4,32) e residentes na Região Nordeste $(O R=1,50$; IC95\%: 1,19-1,89). Diversos indicadores de posição socioeconômica influenciam a percepção sobre a saúde bucal, mas a renda domiciliar per capita, a escolaridade e a classe social foram as responsáveis pelo maior gradiente na autoavaliação da saúde bucal de adultos no Brasil em 2013.

Autoavaliação; Saúde Bucal; Disparidades nos Níveis de Saúde; Condições Sociais; Inquéritos Epidemiológicos
Jailson Lopes de Sousa 1,2

Ana Henriques 3

Zilda Pereira da Silva 2

Milton Severo 3

Susana Silva 3

doi: 10.1590/0102-311X00099518

\author{
Correspondência \\ J. L. Sousa \\ Programa de Pós-graduação em Epidemiologia, Faculdade de \\ Saúde Pública, Universidade de São Paulo. \\ Av. Dr. Arnaldo 715, São Paulo, SP 03178-200, Brasil. \\ jailson.l.sousa@ibge.gov.br \\ 1 Instituto Brasileiro de Geografia e Estatística, São Paulo, \\ Brasil. \\ 2 Faculdade de Saúde Pública, Universidade de São Paulo, São \\ Paulo, Brasil. \\ 3 Instituto de Saúde Pública, Universidade do Porto, Portugal.
}




\section{Introdução}

A implantação do Sistema Único de Saúde (SUS), em 1990, e da Política Nacional de Saúde Bucal - Brasil Sorridente, em 2004, ampliou o acesso aos cuidados em saúde bucal na atenção primária e especializada e à água tratada e fluoretada, contribuindo para o fortalecimento das ações de promoção e prevenção em saúde bucal no Brasil 1,2,3,4. Essas iniciativas, por sua vez, resultaram na melhoria das condições de saúde bucal da população brasileira, ilustrada pela condição de prevalência de cárie, que mudou de moderada, em 2003, para baixa em 2010, segundo critério adotado pela Organização Mundial da Saúde (OMS) 4. Porém, a carga de doenças e os impactos que comprometem a qualidade de vida dos indivíduos ainda permanecem elevados, sobretudo nos grupos sociais menos favorecidos 1,5,6,7,8.

De fato, a autoavaliação da saúde bucal tende a piorar entre os indivíduos menos escolarizados 9,10,11, com menor renda 6,12,13, pertencentes a classes sociais menos privilegiadas 9,10,14, moradores de regiões economicamente menos desenvolvidas 1,5 e que se declaram não brancos 1,5,7. Já os resultados quanto à influência da idade e do sexo não são consensuais: alguns estudos indicam que a autoavaliação da saúde bucal é pior entre os mais velhos 7,9,15,16 e entre as mulheres 6,7, outros mostram que os mais velhos referem melhor saúde bucal 5,17,18, ao passo que algumas pesquisas não encontram associação entre a idade 19,20 ou o sexo 5,20,21,22 e a autoavaliação de saúde bucal. Adicionalmente, apesar de a classe social constituir um indicador adequado para dar conta da heterogeneidade da estrutura socioeconômica de países como o Brasil 23, são escassos os estudos nacionais que analisam a sua influência sobre a autoavaliação da saúde bucal ${ }^{19}$. Importa, assim, avaliar em que medida a classe social representa um indicador válido para captar diferenças na autoavaliação da saúde bucal, no contexto de outros marcadores de um sistema de vigilância de equidade em saúde (como sexo, idade, cor ou raça, região de residência, escolaridade e renda) 24 e medidas objetivas de saúde (como perda dentária, condições clínicas de saúde bucal, frequência de consulta ao dentista, higiene bucal e tabagismo) 5,15,21,25,26.

Logo, o objetivo deste estudo foi analisar a autoavaliação da saúde bucal da população adulta brasileira segundo a posição socioeconômica, explorando as variáveis com maior sensibilidade para medir tal associação.

\section{Métodos}

\section{Desenho de estudo e participantes}

Foram utilizados os microdados da Pesquisa Nacional de Saúde (PNS) 2013, um inquérito domiciliar de base populacional, realizado pelo Instituto Brasileiro de Geografia e Estatística (IBGE) em parceria com o Ministério da Saúde, previsto para ocorrer a cada cinco anos. A PNS foi aprovada pela Comissão Nacional de Ética em Pesquisa, do Conselho Nacional de Saúde, em junho de 2013 (no 10853812.7.0000.0008) 27.

O trabalho de campo ocorreu entre agosto de 2013 e fevereiro de 2014, e foram realizadas entrevistas face a face por agentes de pesquisa do IBGE devidamente treinados e com o auxílio de um computador de mão (Personal Digital Assistant). Os participantes da pesquisa eram moradores de domicílios particulares permanentes, com área de abrangência em todo território nacional.

Os dados da PNS foram obtidos com base em um plano amostral complexo, já descrito anteriormente 28 , englobando conglomerados com três estágios de seleção: setor censitário ou conjunto de setores censitários (unidades primárias de amostragem), domicílios particulares permanentes (unidades secundárias) e um morador com 18 ou mais anos de idade por domicílio (unidades terciárias). Um total de 69.994 moradores adultos selecionados foram convidados a participar, sendo que 60.202 (86\%) foram entrevistados. A amostra final deste estudo compreendeu 59.758 participantes com informação completa para todas as variáveis utilizadas, após a exclusão dos que não apresentavam informação para cor ou raça $(n=3)$ e dos que apenas ajudavam, sem remuneração, no trabalho de algum membro do domicílio $(n=441)$, que não puderam ser classificados na tipologia de classe social utilisada nem considerados como pessoas fora da força de trabalho. 


\section{Variáveis de estudo}

A autoavaliação da saúde bucal foi estudada por meio da seguinte questão: "Em geral, como o(a) senhor(a) avalia sua saúde bucal (dentes e gengivas)?”, com cinco opções de respostas. Essas foram agregadas em positiva (incluindo as categorias "muito boa" e "boa"), regular e negativa (incluindo as categorias "ruim" e "muito ruim").

Para definir a posição socioeconômica foram utilizadas as seguintes variáveis: região de residência (Sul, Sudeste, Centro-oeste, Norte e Nordeste), nível de escolaridade completo (Ensino Superior, Ensino Médio, Ensino Fundamental e nenhum), renda domiciliar per capita em salários mínimos ( $>6$, $>3-6,>1-3$ e $\leq 1)$ e classe social. A renda domiciliar per capita resultou da soma dos rendimentos brutos mensais em dinheiro e produtos de todos os indivíduos do domicílio, dividida pelo número total de moradores. A classe social foi definida com base na tipologia proposta por Santos para o Brasil 23, cruzando informações da posição na ocupação, o grupo ocupacional (baseando-se na Classificação de Ocupações para Pesquisas Domiciliares - COD 29) e a renda individual. Essa tipologia contempla 15 posições de classe, podendo ser agregadas nos seguintes grupos: posições privilegiadas (capitalistas e fazendeiros, especialistas autônomos, gerentes, empregados especialistas e pequenos empregadores); controladores de ativos de menor valor (autônomos com ativos e autônomos agrícolas); classe trabalhadora não destituída (empregados qualificados, supervisores e trabalhadores típicos) e destituídos de ativos (trabalhadores elementares, autônomos precários, empregados domésticos, agrícolas precários e trabalhadores excedentes). Seguindo a proposta de Rose \& O’Reilly 30, a categoria “pessoas fora da força de trabalho" foi adicionada, a qual compreende pessoas que não trabalhavam nem procuravam emprego no mês de referência da pesquisa, segundo o IBGE 31 .

Como variáveis que poderiam influenciar a associação entre a autoavaliação da saúde bucal e os diversos indicadores de posição socioeconômica, utilizaram-se o sexo (feminino e masculino), a idade (18-29, 30-44, 45-59 e $\geq 60$ anos), a cor ou raça autodeclarada (branca, parda, preta e outras), a dentição funcional (presença de 20 ou mais dentes naturais 32), a dificuldade de se alimentar devido a problemas nos dentes ou dentadura, consultar o dentista nos últimos 12 meses, escovar os dentes 2 ou mais vezes por dia e o tabagismo (nunca fumou, ex-fumante ou fumante).

As variáveis do estudo foram selecionadas à luz do modelo teórico de determinação da autoavaliação da saúde bucal proposto por Martins et al. 5 , segundo o qual a forma como as pessoas percebem sua saúde bucal é definida por aspectos do ambiente externo, características individuais, comportamentos relacionados à saúde, condições objetivas de saúde e condições subjetivas relacionadas à saúde.

\section{Análise de dados}

A análise foi realizada no software Stata versão 11.0 (https://www.stata.com) com o módulo survey, levando-se em consideração todos os elementos que definem uma amostragem complexa 33, tais como a estratificação, a conglomeração, as probabilidades desiguais de seleção e o peso amostral para o morador adulto selecionado, com correção de não entrevista e calibração pela projeção da população. Foram calculadas frequências absolutas e relativas de todas as variáveis de estudo.

Para avaliar a associação entre a posição socioeconômica e autoavaliação da saúde bucal foram calculados odds ratio (OR) e os respectivos intervalos de 95\% de confiança (IC95\%) por meio de modelos de regressão logística multinomial. Para cada variável de posição socioeconômica considerada foram construídos modelos independentes, brutos e ajustados para todas as variáveis que mostraram confundir a associação entre posição socioeconômica e autoavaliação da saúde bucal.

Para averiguar qual dos modelos explicaria melhor o desfecho em questão, foram calculados os porcentuais de concordância absoluta e o valor de kappa de Cohen para comparar os valores obtidos pelos modelos e o esperado tão somente devido ao acaso ${ }^{34}$. Os indivíduos foram classificados na classe modal em termos de probabilidades estimadas pelo modelo. 


\section{Resultados}

A Tabela 1 mostra as características da população em estudo de acordo com as categorias da autoavaliação da saúde bucal. A maior proporção de indivíduos era do sexo feminino, com idades entre 30 e 44 anos, de cor ou raça branca, residente no Sudeste brasileiro, não apresentava qualquer nível de escolaridade completo, possuía renda domiciliar per capita menor ou igual a um salário mínimo e encontrava-se fora da força de trabalho. A maioria das pessoas possuía dentição funcional, não apresentava qualquer dificuldade de se alimentar devido a problemas nos dentes ou dentadura e escovava os dentes duas ou mais vezes por dia. No entanto, pouco mais da metade referiu não ter ido ao dentista nos últimos 12 meses. Mais de dois terços dos indivíduos nunca fumaram.

Tabela 1

Caracterização da população de estudo ( $n=59.758)$ segundo variáveis investigadas e categorias da autoavaliação da saúde bucal. Brasil, 2013.

\begin{tabular}{|c|c|c|c|c|}
\hline \multirow[t]{3}{*}{ Variáveis } & \multicolumn{4}{|c|}{$\%$ com peso amostral } \\
\hline & \multirow[t]{2}{*}{ Na amostra total } & \multicolumn{3}{|c|}{ Autoavaliação da saúde bucal } \\
\hline & & Positiva & Regular & Negativa \\
\hline \multicolumn{5}{|l|}{ Sexo } \\
\hline Feminino & 52,7 & 68,9 & 25,4 & 5,7 \\
\hline Masculino & 47,3 & 65,8 & 28,2 & 6,1 \\
\hline \multicolumn{5}{|l|}{ Idade (anos) } \\
\hline $18-29$ & 26,0 & 74,0 & 23,0 & 3,1 \\
\hline $30-44$ & 30,7 & 68,6 & 26,7 & 4,7 \\
\hline $45-59$ & 25,2 & 62,9 & 28,5 & 8,6 \\
\hline$\geq 60$ & 18,1 & 62,3 & 29,7 & 8,0 \\
\hline \multicolumn{5}{|c|}{ Cor ou raça (autodeclarada) } \\
\hline Branca & 47,5 & 73,1 & 22,2 & 4,7 \\
\hline Parda & 41,9 & 62,5 & 30,7 & 6,8 \\
\hline Preta & 9,2 & 61,1 & 31,1 & 7,8 \\
\hline Outras * & 1,4 & 63,7 & 31,9 & 4,4 \\
\hline \multicolumn{5}{|l|}{ Região de residência } \\
\hline Sul & 14,7 & 72,1 & 22,6 & 5,3 \\
\hline Sudeste & 44,0 & 72,1 & 23,1 & 4,7 \\
\hline Centro-oeste & 7,4 & 68,8 & 26,1 & 5,1 \\
\hline Norte & 7,4 & 59,7 & 34,4 & 6,0 \\
\hline Nordeste & 26,5 & 58,8 & 32,9 & 8,3 \\
\hline \multicolumn{5}{|l|}{ Escolaridade (completa) } \\
\hline Ensino Superior & 12,8 & 83,5 & 14,6 & 2,0 \\
\hline Ensino Médio & 32,9 & 73,3 & 23,5 & 3,3 \\
\hline Ensino Fundamental & 15,5 & 66,8 & 28,1 & 5,2 \\
\hline Nenhum & 38,8 & 57,5 & 32,9 & 9,6 \\
\hline \multicolumn{5}{|c|}{ Renda domiciliar per capita ** (em salários mínimos) } \\
\hline$>6$ & 4,2 & 88,3 & 10,3 & 1,3 \\
\hline$>3-6$ & 8,0 & 82,5 & 15,7 & 1,8 \\
\hline$>1-3$ & 38,2 & 71,8 & 23,9 & 4,3 \\
\hline$\leq 1$ & 46,6 & 59,9 & 32,0 & 8,1 \\
\hline
\end{tabular}

(continua) 


\begin{tabular}{|c|c|c|c|c|}
\hline \multirow[t]{3}{*}{ Variáveis } & \multicolumn{4}{|c|}{$\%$ com peso amostral } \\
\hline & \multirow[t]{2}{*}{ Na amostra total } & \multicolumn{3}{|c|}{ Autoavaliação da saúde bucal } \\
\hline & & Positiva & Regular & Negativa \\
\hline \multicolumn{5}{|l|}{ Classe social } \\
\hline Posições privilegiadas & 8,6 & 83,3 & 14,5 & 2,2 \\
\hline Controladores de ativos de menor valor & 6,3 & 67,0 & 27,5 & 5,5 \\
\hline Classe trabalhadora não destituída & 28,5 & 73,0 & 23,7 & 3,2 \\
\hline Destituídos de ativos & 21,4 & 60,4 & 31,8 & 7,8 \\
\hline Pessoas fora da força de trabalho & 35,3 & 63,4 & 28,8 & 7,8 \\
\hline \multicolumn{5}{|l|}{ Dentição funcional *** } \\
\hline $\operatorname{Sim}$ & 86,5 & 67,9 & 26,8 & 5,2 \\
\hline Não & 13,5 & 64,3 & 25,9 & 9,9 \\
\hline \multicolumn{5}{|c|}{ Dificuldade de se alimentar devido a problemas nos dentes/dentadura } \\
\hline $\operatorname{Sim}$ & 10,4 & 28,4 & 45,9 & 25,7 \\
\hline Não & 89,6 & 72,0 & 24,5 & 3,6 \\
\hline \multicolumn{5}{|l|}{ Consulta ao dentista nos últimos 12 meses } \\
\hline Sim & 44,4 & 74,2 & 22,0 & 3,7 \\
\hline Não & 55,6 & 62,0 & 30,4 & 7,6 \\
\hline \multicolumn{5}{|l|}{ Escova os dentes 2 ou mais vezes/dia } \\
\hline Sim & 90,9 & 69,7 & 25,6 & 4,7 \\
\hline Não & 9,1 & 48,3 & 37,2 & 14,6 \\
\hline \multicolumn{5}{|l|}{ Tabagismo } \\
\hline Nunca fumou & 67,8 & 71,1 & 24,5 & 4,3 \\
\hline Ex-fumante & 17,5 & 62,5 & 30,0 & 7,5 \\
\hline Fumante & 14,7 & 56,4 & 32,7 & 10,9 \\
\hline
\end{tabular}

* Inclui "amarelo" e "indígena";

** O salário mínimo vigente no Brasil em 2013 era de $\mathrm{R} \$$ 678,00 48;

*** Dentição funcional: indivíduos com $\geq 20$ dentes naturais 32 .

Considerando a população total, mais de dois terços dos indivíduos avaliaram positivamente a sua saúde bucal (9,9\% muito boa e 57,5\% boa), 26,7\% consideraram-na regular e 5,9\% negativa (4,9\% ruim e $1 \%$ muito ruim).

Analisando a autoavaliação da saúde bucal segundo as variáveis de posição socioeconômica investigadas, as proporções das condições regular e negativa foram maiores entre os residentes no Norte e Nordeste, com menor escolaridade, menor renda domiciliar per capita, das classes sociais menos privilegiadas e entre os indivíduos que declararam possuir as piores condições e cuidados em saúde bucal, e entre os fumantes (Tabela 1).

Considerando cada indicador de posição socioeconômica de forma independente, a autoavaliação da saúde bucal tendeu a piorar com os menores estratos de escolaridade e de renda domiciliar per capita. As pessoas em posições de classe não privilegiada e moradoras do Norte ou Nordeste revelaram-se estatisticamente mais propensas a avaliar sua saúde bucal como regular ou negativa (Tabela 2).

Nas Tabelas 3 e 4 são apresentados os modelos resultantes da regressão múltipla da associação entre a posição socioeconômica e a autoavaliação da saúde bucal. De modo a identificar qual variável alusiva à posição socioeconômica seria mais sensível para analisar a autoavaliação da saúde bucal, foram construídos modelos englobando cada uma das variáveis de posição socioeconômica separadamente. Assim, após ajuste para as variáveis confundidoras já referidas, os indicadores de posição socioeconômica mais fortemente associados com maiores gradientes na autoavaliação da saúde bucal foram: a renda domiciliar per capita, a escolaridade e a classe social. Pessoas com até um salário mínimo per capita (OR = 3,59; IC95\%: 2,85-4,54), seguidas dos indivíduos com nenhum nível de escolaridade completa $(\mathrm{OR}=2,40$; IC95\%: 2,10-2,74) e daqueles pertencentes à classe social destituídos 
Tabela 2

Odds ratios (OR) brutos e respectivos intervalos de 95\% de confiança (IC95\%) para a associação entre posição socioeconômica e autoavaliação da saúde bucal. Brasil, 2013.

\begin{tabular}{|c|c|c|c|c|c|}
\hline \multirow[t]{3}{*}{ Variáveis } & \multicolumn{5}{|c|}{ Autoavaliação da saúde bucal } \\
\hline & \multirow[t]{2}{*}{ Positiva } & \multicolumn{2}{|c|}{ Regular } & \multicolumn{2}{|c|}{ Negativa } \\
\hline & & OR & IC95\% & OR & IC95\% \\
\hline \multicolumn{6}{|l|}{ Região de residência } \\
\hline Sul & 1,00 & 1,00 & & 1,00 & \\
\hline Sudeste & 1,00 & 1,02 & $0,91-1,15$ & 0,90 & $0,72-1,13$ \\
\hline Centro-oeste & 1,00 & 1,21 & $1,08-1,36$ & 1,02 & $0,81-1,28$ \\
\hline Norte & 1,00 & 1,84 & $1,63-2,07$ & 1,37 & $1,07-1,75$ \\
\hline Nordeste & 1,00 & 1,79 & $1,60-1,99$ & 1,92 & $1,56-2,36$ \\
\hline \multicolumn{6}{|l|}{ Escolaridade (completa) } \\
\hline Ensino Superior & 1,00 & 1,00 & & 1,00 & \\
\hline Ensino Médio & 1,00 & 1,84 & $1,62-2,08$ & 1,86 & $1,33-2,61$ \\
\hline Ensino Fundamental & 1,00 & 2,41 & $2,11-2,75$ & 3,23 & $2,32-4,49$ \\
\hline Nenhum & 1,00 & 3,28 & $2,90-3,72$ & 7,00 & $5,20-9,42$ \\
\hline \multicolumn{6}{|c|}{ Renda domiciliar per capita * (em salários mínimos) } \\
\hline$>6$ & 1,00 & 1,00 & & 1,00 & \\
\hline$>3-6$ & 1,00 & 1,63 & $1,28-2,07$ & 1,40 & $0,77-2,52$ \\
\hline$>1-3$ & 1,00 & 2,85 & $2,27-3,57$ & 3,91 & $2,34-6,54$ \\
\hline$\leq 1$ & 1,00 & 4,57 & $3,66-5,71$ & 8,97 & $5,45-14,76$ \\
\hline \multicolumn{6}{|l|}{ Classe social } \\
\hline Posições privilegiadas & 1,00 & 1,00 & & 1,00 & \\
\hline Controladores de ativos de menor valor & 1,00 & 2,35 & $1,94-2,85$ & 3,08 & $2,07-4,57$ \\
\hline Classe trabalhadora não destituída & 1,00 & 1,86 & $1,58-2,19$ & 1,66 & $1,17-2,35$ \\
\hline Destituídos de ativos & 1,00 & 3,02 & $2,58-3,54$ & 4,84 & $3,46-6,77$ \\
\hline Pessoas fora da força de trabalho & 1,00 & 2,61 & $2,24-3,04$ & 4,58 & $3,27-6,39$ \\
\hline
\end{tabular}

* O salário mínimo vigente no Brasil em 2013 era de $\mathrm{R} \$ 678,0048$.

de ativos (OR = 2,38; IC95\%: 2,03-2,80) revelaram-se significativamente mais propensas a autoavaliar sua saúde bucal como regular, em detrimento de avaliações positivas (Tabela 3). As mesmas condições se mostraram importantes para a autoavaliação negativa, ainda que com razões de chances distintas: a renda domiciliar per capita de até um salário mínimo destacou-se $(\mathrm{OR}=4,71$; IC95\%: 2,84-7,83), seguida da ausência de instrução $(\mathrm{OR}=3,28$; IC95\%: 2,34-4,61) e dos destituídos de ativos $(\mathrm{OR}=3,03$; IC95\%: 2,12-4,32) (Tabela 4).

Todos os modelos de regressão (Tabelas 3 e 4) obtiveram resultados semelhantes em relação à concordância (valor de kappa) entre o modelo de regressão obtido e o esperado tão somente devido ao acaso.

\section{Discussão}

Os resultados deste estudo mostram que mais de dois terços da população adulta brasileira avaliam positivamente sua saúde bucal e que diversos indicadores socioeconômicos podem influenciar esta autoavaliação. Dos marcadores considerados, uma posição socioeconômica mais desfavorável está associada à percepção mais negativa da saúde bucal, de modo que a renda domiciliar per capita mais baixa, a ausência de escolaridade e o pertencimento à classe social destituída de ativos estão associados a uma pior autoavaliação da saúde bucal.

Estudos internacionais, valendo-se de inquéritos populacionais com adultos, indicaram prevalências diversas de saúde bucal positiva: 83\% na Austrália entre 2004-2006 17, 81,7\% em Toronto/ 


\section{Tabela 3}

Odds ratios (OR) ajustados e respectivos intervalos de 95\% de confiança (IC95\%) para a associação entre posição socioeconômica e autoavaliação da saúde bucal (regular versus positiva). Brasil, 2013.

\begin{tabular}{|c|c|c|c|c|c|c|c|c|}
\hline \multirow[t]{2}{*}{ Variáveis } & \multicolumn{2}{|c|}{ Modelo 1} & \multicolumn{2}{|c|}{ Modelo 2} & \multicolumn{2}{|c|}{ Modelo 3} & \multicolumn{2}{|c|}{ Modelo 4} \\
\hline & OR & IC95\% & OR & IC95\% & OR & IC95\% & OR & IC95\% \\
\hline \multicolumn{9}{|l|}{ Região de residência } \\
\hline Sul & 1,00 & & - & - & - & - & - & - \\
\hline Sudeste & 0,99 & $0,88-1,11$ & - & - & - & - & - & - \\
\hline Centro-oeste & 1,10 & $0,97-1,24$ & - & - & - & - & - & - \\
\hline Norte & 1,56 & $1,37-1,78$ & - & - & - & - & - & - \\
\hline Nordeste & 1,47 & $1,31-1,65$ & - & - & - & - & - & - \\
\hline \multicolumn{9}{|l|}{ Escolaridade (completa) } \\
\hline Ensino Superior & - & - & 1,00 & & - & - & - & - \\
\hline Ensino Médio & - & - & 1,75 & $1,55-1,98$ & - & - & - & - \\
\hline Ensino Fundamental & - & - & 2,09 & $1,83-2,38$ & - & - & - & - \\
\hline Nenhum & - & - & 2,40 & $2,10-2,74$ & - & - & - & - \\
\hline \multirow{2}{*}{\multicolumn{9}{|c|}{$\begin{array}{l}\text { Renda domiciliar per capita (em salários } \\
\text { mínimos) }\end{array}$}} \\
\hline & & & & & & & & \\
\hline$>6$ & - & - & - & - & 1,00 & & - & - \\
\hline$>3-6$ & - & - & - & - & 1,53 & $1,20-1,95$ & - & - \\
\hline$>1-3$ & - & - & - & - & 2,48 & $1,97-3,14$ & - & - \\
\hline$\leq 1$ & - & - & - & - & 3,59 & $2,85-4,54$ & - & - \\
\hline \multicolumn{9}{|l|}{ Classe social } \\
\hline Posições privilegiadas & - & - & - & - & - & - & 1,00 & \\
\hline Controladores de ativos de menor valor & - & - & - & - & - & - & 1,96 & $1,61-2,38$ \\
\hline Classe trabalhadora não destituída & - & - & - & - & - & - & 1,73 & $1,48-2,04$ \\
\hline Destituídos de ativos & - & - & - & - & - & - & 2,38 & $2,03-2,80$ \\
\hline Pessoas fora da força de trabalho & - & - & - & - & - & - & 2,18 & $1,86-2,55$ \\
\hline \% de concordância (valor de kappa) & \multicolumn{2}{|c|}{$67,6(0,16)$} & \multicolumn{2}{|c|}{$67,8(0,16)$} & \multicolumn{2}{|c|}{$67,7(0,16)$} & \multicolumn{2}{|c|}{$67,7(0,16)$} \\
\hline
\end{tabular}

Modelo 1: região de residência + ajuste para sexo, idade, cor ou raça, dificuldade de se alimentar devido a problemas nos dentes/dentadura, dentição funcional, consulta ao dentista nos últimos 12 meses, escova os dentes 2 ou mais vezes/ dia e tabagismo;

Modelo 2: nível de escolaridade + ajuste para sexo, idade, cor ou raça, dificuldade de se alimentar devido a problemas nos dentes/dentadura, dentição funcional, consulta ao dentista nos últimos 12 meses, escova os dentes 2 ou mais vezes/ dia e tabagismo;

Modelo 3: renda domiciliar per capita + ajuste para sexo, idade, cor ou raça, dificuldade de se alimentar devido a problemas nos dentes/dentadura, dentição funcional, consulta ao dentista nos últimos 12 meses, escova os dentes 2 ou mais vezes/dia e tabagismo;

Modelo 4: classe social + ajuste para sexo, idade, cor ou raça, dificuldade de se alimentar devido a problemas nos dentes/dentadura, dentição funcional, consulta ao dentista nos últimos 12 meses, escova os dentes 2 ou mais vezes/dia e tabagismo.

* O salário mínimo vigente no Brasil em 2013 era de $\mathrm{R} \$ 678,0048$.

Canadá em 2003 26, 75\% na Suécia em 2012 15, 68,6\% nos Estados Unidos (2005-2008) e 69,2\% na Inglaterra (2009) 11, contrastando com 35,2\% em Istambul, Turquia em 2003 35. Estudos nacionais encontraram prevalências da autopercepção positiva da saúde entre adultos de 60,9\% nos moradores de quatro municípios do Estado do Rio de Janeiro em 2005 6, 66,8\% no Município de Florianópolis, Santa Catarina, Brasil em 2009 7, 44,2\% entre indivíduos de 35-44 anos residentes na Região Sudeste do Brasil em 200336 e 37\% entre residentes no nordeste brasileiro em 2010 37. Os resultados da Pesquisa Nacional de Saúde Bucal - SBBrasil 20104 mostraram que 40,3\% dos brasileiros de 35-44 anos de idade tinham uma percepção positiva da sua saúde bucal, variando entre as regiões: de 36,1\% no 


\section{Tabela 4}

Odds ratios (OR) ajustados e respectivos intervalos de 95\% de confiança (IC95\%) para a associação entre posição socioeconômica e autoavaliação da saúde bucal (negativa versus positiva). Brasil, 2013.

\begin{tabular}{|c|c|c|c|c|c|c|c|c|}
\hline \multirow[t]{2}{*}{ Variáveis } & \multicolumn{2}{|c|}{ Modelo 1} & \multicolumn{2}{|c|}{ Modelo 2} & \multicolumn{2}{|c|}{ Modelo 3} & \multicolumn{2}{|c|}{ Modelo 4} \\
\hline & OR & IC95\% & OR & IC95\% & OR & IC95\% & OR & IC95\% \\
\hline \multicolumn{9}{|l|}{ Região de residência } \\
\hline Sul & 1,00 & & - & - & - & - & - & - \\
\hline Sudeste & 0,95 & $0,75-1,21$ & - & - & - & - & - & - \\
\hline Centro-oeste & 0,95 & $0,73-1,23$ & - & - & - & - & - & - \\
\hline Norte & 1,15 & $0,87-1,52$ & - & - & - & - & - & - \\
\hline Nordeste & 1,50 & $1,19-1,89$ & - & - & - & - & - & - \\
\hline \multicolumn{9}{|l|}{ Escolaridade (completa) } \\
\hline Ensino Superior & - & - & 1,00 & & - & - & - & - \\
\hline Ensino Médio & - & - & 1,83 & $1,29-2,61$ & - & - & - & - \\
\hline Ensino Fundamental & - & - & 2,50 & $1,76-3,55$ & - & - & - & - \\
\hline Nenhum & - & - & 3,28 & $2,34-4,61$ & - & - & - & - \\
\hline \multicolumn{9}{|c|}{ Renda domiciliar per capita *(em salários mínimos) } \\
\hline$>6$ & - & - & - & - & 1,00 & & - & - \\
\hline$>3-6$ & - & - & - & - & 1,12 & $0,62-2,04$ & - & - \\
\hline$>1-3$ & - & - & - & - & 2,58 & $1,54-4,32$ & - & - \\
\hline$\leq 1$ & - & - & - & - & 4,71 & $2,84-7,83$ & - & - \\
\hline \multicolumn{9}{|l|}{ Classe social } \\
\hline Posições privilegiadas & - & - & - & - & - & - & 1,00 & \\
\hline Controladores de ativos de menor valor & - & - & - & - & - & - & 2,15 & $1,43-3,24$ \\
\hline Classe trabalhadora não destituída & - & - & - & - & - & - & 1,53 & $1,06-2,19$ \\
\hline Destituídos de ativos & - & - & - & - & - & - & 3,03 & $2,12-4,32$ \\
\hline Pessoas fora da força de trabalho & - & - & - & - & - & - & 2,75 & $1,91-3,96$ \\
\hline \% de concordância (valor de kappa) & \multicolumn{2}{|c|}{$67,6(0,16)$} & \multicolumn{2}{|c|}{$67,8(0,16)$} & \multicolumn{2}{|c|}{$67,7(0,16)$} & \multicolumn{2}{|c|}{$67,7(0,16)$} \\
\hline
\end{tabular}

Modelo 1: região de residência + ajuste para sexo, idade, cor ou raça, dificuldade de se alimentar devido a problemas nos dentes/dentadura, dentição funcional, consulta ao dentista nos últimos 12 meses, escova os dentes 2 ou mais vezes/dia e tabagismo;

Modelo 2: nível de escolaridade + ajuste para sexo, idade, cor ou raça, dificuldade de se alimentar devido a problemas nos dentes/dentadura, dentição funcional, consulta ao dentista nos últimos 12 meses, escova os dentes 2 ou mais vezes/dia e tabagismo;

Modelo 3: renda domiciliar per capita + ajuste para sexo, idade, cor ou raça, dificuldade de se alimentar devido a problemas nos dentes/dentadura, dentição funcional, consulta ao dentista nos últimos 12 meses, escova os dentes 2 ou mais vezes/dia e tabagismo;

Modelo 4: classe social + ajuste para sexo, idade, cor ou raça, dificuldade de se alimentar devido a problemas nos dentes/dentadura, dentição funcional, consulta ao dentista nos últimos 12 meses, escova os dentes 2 ou mais vezes/dia e tabagismo.

* O salário mínimo vigente no Brasil em 2013 era de $\mathrm{R} \$ 678,0048$.

Norte a 47,3\% no Sul do país. Essa mesma prevalência foi de 52,6\% para aqueles entre 65-74 anos de idade, variando de 47,9\% no Norte a 58,2\% no Centro-oeste. Essas diferenças podem ser resultado de desigualdades no acesso aos serviços de saúde, bem como de distintas percepções acerca do estado de saúde e de estilos de vida, influenciadas por valores sociais, culturais e emocionais e pelo bem-estar individual 6,7 .

Os achados deste estudo mostram que as chances de os indivíduos avaliarem sua saúde bucal de forma menos favorável aumentam na medida em que diminuem a renda domiciliar per capita e a escolaridade, corroborando os resultados de pesquisas anteriores 6,7,10,11,12,13,19. Indivíduos economicamente desfavorecidos tendem a sofrer maiores impactos negativos dos problemas com seus dentes, boca ou próteses dentárias e relatar maior perda de elementos dentários do que os indivíduos em melhores condições econômicas ${ }^{7}$. Já as pessoas mais escolarizadas apresentam maior envolvimento no autocuidado e buscam mais frequentemente a resolução de problemas de saúde, além de usufruí- 
rem de melhores condições sociais e econômicas, como emprego, renda, posse de bens e acesso a serviços $1,5,10,38,39,40$. O papel de destaque da renda como um indicador de posição socioeconômica fortemente associado aos maiores gradientes de desigualdade na autopercepção da saúde bucal da população brasileira traz implicações para as políticas de saúde, pois aponta a necessidade de reforçar o acesso a cuidados de saúde bucal nos grupos mais desfavorecidos em termos econômicos.

Os resultados aqui analisados corroboram achados de outras investigações 10,11,15,20,25, ao mostrarem que as chances de autoavaliar a saúde bucal como regular e negativa são maiores entre pessoas com posições de classe menos privilegiadas (destituídos de ativos, pessoas fora da força de trabalho, controladores de ativos de menor valor e classe trabalhadora não destituída de ativos). Desse modo, contribuem para o debate em torno da classe social como indicador de posição socioeconômica relevante em estudos de base populacional no Brasil 41 , sustentando o investimento na coleta sistemática de dados que permitam obter informações acerca da ocupação e condição de emprego. A exclusão do mercado de trabalho tem efeitos deletérios para a saúde, assim como a natureza extenuante, cansativa e arriscada de algumas atividades profissionais, bem como a ausência de regulamentação dos contratos de trabalho. A tipologia original de classe proposta por Figueiredo Santos foi validada para o Brasil 42 e sua adaptação metodológica 23 , utilizada no presente estudo, não exige informações sobre o número de empregados, a propriedade do estabelecimento e o tamanho da propriedade agrícola, variáveis que são importantes na proposta original do autor, mas que não foram recolhidas no âmbito da PNS 2013.

Ainda que a renda, a escolaridade e a classe social apareçam como fatores socioeconômicos mais relevantes para a autoavaliação da saúde bucal na população adulta brasileira, este estudo reforça a importância de incluir outras variáveis no monitoramento das desigualdades sociais em saúde também contempladas pela OMS, como local de residência, sexo, idade e cor ou raça 43.

Os residentes do Norte e Nordeste tenderam a avaliar pior sua condição de saúde bucal. Esses resultados são reflexos da heterogeneidade regional que persiste ao longo da história no país, compondo uma polaridade e separação em dois blocos: Norte/Nordeste e Sul/Sudeste/Centro-oeste 44, com o primeiro bloco marcado pelas regiões com as piores condições sociais, de saúde e de acesso aos serviços de saúde no Brasil 1,2,4,5,44.

Homens e mulheres parecem não diferir significativamente quanto à autoavaliação da saúde bucal 5,19,20,21. Embora as mulheres utilizem mais os serviços de saúde e expressem mais a existência de morbidades 19,41, geralmente há maior prevalência de comportamentos associados com a autoavaliação negativa da saúde bucal entre os homens, tais como hábito de fumar e menor frequência de escovação dos dentes $1,15,42$.

Destaca-se na literatura que as maiores probabilidades de autoavaliar negativamente a saúde bucal são encontradas nas faixas intermediárias de 40-60 anos 6,14,15,19. Esse comportamento suporta a ideia de que as desigualdades na saúde bucal subjetiva se ampliam no início e em meados da vida adulta e tendem a diminuir nas idades mais avançadas 10 , não só porque os idosos naturalizam algumas dores e incapacidades como processos inevitáveis do envelhecimento, como também porque acreditam haver real melhora da condição bucal na substituição dos dentes naturais por próteses parciais ou totais $5,6,18,19$.

Em relação à cor ou raça, as evidências apontam que os indivíduos que se declaram brancos geralmente apresentam condições de vida mais favoráveis 1,5,45. Uma análise da estratificação social de cor no Brasil destaca haver um contraste marcante em termos de recompensas materiais entre brancos e negros (pretos e pardos), com reflexos num ciclo acumulativo de desvantagens que afeta a trajetória de vida dos indivíduos negros, colocando-os em posições inferiores na hierarquia social, com repercussões no seu estado de saúde 45 .

Este estudo apresenta algumas limitações que precisam ser discutidas. Por se tratar de um estudo transversal não permite estabelecer uma relação temporal entre as associações encontradas, o que limita, até certo ponto, a interpretação dos resultados. Todas as informações foram autorreferidas, não havendo a realização de exames clínicos sobre a saúde bucal, e a literatura reconhece haver diferenças entre avaliações de saúde realizadas por profissionais e aquelas feitas pelos próprios indivíduos 46. Porém, realçar a subjetividade nas avaliações da saúde é encarada como aporte crítico essencial aos indicadores clínicos utilizados pelos profissionais de saúde, auxiliando no refinamento dos diagnósticos e na identificação de pessoas ou grupos populacionais em situação de vulnerabilidade 20,38,47. 
Já a principal força do estudo foi usar um banco de dados de abrangência nacional com um processo de amostragem probabilística. Isso possibilita generalizar os resultados, além de proporcionar a análise do desfecho em três categorias. Adicionalmente, permite avaliar com maior nuance o efeito dos indicadores de posição socioeconômica na desigualdade da autoavaliação da saúde bucal.

Sabendo-se que a forma como os indivíduos percebem sua saúde influencia a procura por serviços de saúde e a incorporação de medidas de autocuidado, a compreensão da relação dos indicadores de posição socioeconômica com esta percepção pode contribuir para o melhor direcionamento de políticas públicas para grupos sociais mais prioritários, contribuindo para a redução das iniquidades em saúde bucal que persistem no Brasil.

\section{Colaboradores}

J. L. Sousa contribuiu na concepção e projeto, análise e interpretação dos dados, redação e revisão crítica do artigo, aprovação da versão final do manuscrito e responsabilidade por todos os aspectos do trabalho na garantia da integralidade de qualquer parte da obra. A. Henriques e S. Silva contribuíram na concepção e projeto, análise e interpretação dos dados, revisão crítica do artigo, aprovação da versão final do manuscrito e responsabilidade por todos os aspectos do trabalho na garantia da integralidade de qualquer parte da obra. Z. P. Silva colaborou na análise e interpretação dos dados, revisão crítica do artigo, aprovação da versão final do manuscrito e responsabilidade por todos os aspectos do trabalho na garantia da integralidade de qualquer parte da obra. M. Severo contribuiu na análise e interpretação dos dados, aprovação da versão final do manuscrito e responsabilidade por todos os aspectos do trabalho na garantia da integralidade de qualquer parte da obra.

\section{Informações adicionais}

ORCID: Jailson Lopes de Sousa (0000-0003-18721765); Ana Henriques (0000-0001-9484-1887); Zilda Pereira da Silva (0000-0003-4648-113X); Milton Severo (0000-0002-5787-4871); Susana Silva (0000-0002-1335-8648).

\section{Agradecimentos}

J. L. Sousa foi bolsista do Coordenação de Aperfeiçoamento de Pessoal Nível Superior (Capes)/ Programa de Doutorado Sanduíche no Exterior/ Processo no 88881.134588/2016-01.

\section{Referências}

1. Nico LS, Andrade SSCA, Malta DC, Pucca Júnior GA, Peres MA. Saúde bucal autorreferida da população adulta brasileira: resultados da Pesquisa Nacional de Saúde 2013. Ciênc Saúde Colet 2016; 21:389-98.

2. Antunes JLF, Narvai PC. Políticas de saúde bucal no Brasil e seu impacto sobre as desigualdades em saúde. Rev Saúde Pública 2010; 44:360-5.

3. Borges CM, Campos ACV, Vargas AMD, Ferreira EF. Perfil das perdas dentárias em adultos segundo o capital social, características demográficas e socioeconômicas. Ciênc Saúde Colet 2014; 19:1849-58.

4. Ministério da Saúde. SB Brasil 2010 - Pesquisa Nacional de Saúde Bucal: principais resultados. Brasília: Ministério da Saúde; 2012.

5. Martins AMEBL, Barreto SM, Pordeus IA. Auto-avaliação de saúde bucal em idosos: análise com base em modelo multidimensional. Cad Saúde Pública 2009; 25:421-35.

6. Mendonça HLC, Szwarcwald CL, Damacena GN. Autoavaliação de saúde bucal: resultados da Pesquisa Mundial de Saúde - Atenção Básica em quatro municípios do estado do Rio de Janeiro, Brasil, 2005. Cad Saúde Pública 2012; 28:1927-38.

7. Luchi CA, Peres KG, Bastos JL, Peres MA. Desigualdades na autoavaliação de saúde bucal em adultos. Rev Saúde Pública 2013; 47:74051.

8. Costa SM, Martins CC, Bonfim MLC, Zina LG, Paiva SM, Pordeus IA, et al. A systematic review of socioeconomic indicators and dental caries in adults. Int J Public Health Res 2012; 9:3540-74

9. Guarnizo-Herreno CC, Watt RG, Fuller E, Steele JG, Shen J, Morris S, et al. Socioeconomic position and subjective oral health: findings for the adult population in England, Wales and Northern Ireland. BMC Public Health 2014; 14:827-36.

10. Tsakos G, Demakakos P, Breeze E, Watt RG. Social gradients in oral health in older adults: findings from the English longitudinal survey of aging. Am J Public Health 2011; 101:1892-9. 
11. Guarnizo-Herreno CC, Tsakos G, Sheiham A, Marmot MG, Kawachi I, Watt RG. Austin Powers bites back: a cross sectional comparison of US and English national oral health surveys. BMJ 2015; 351:h6543.

12. Brennan DS, Teusner DN. Oral health impacts on self-rated general and oral health in a crosssectional study of working age adults. Community Dent Oral Epidemiol 2015; 43:282-8.

13. Tassinari WS, Léon AP, Werneck GL, Faerstein E, Lopes CS, Chor D, et al. Contexto sócio-econômico e percepção da saúde bucal em uma população de adultos no Rio de Janeiro, Brasil: uma análise multinível. Cad Saúde Pública 2007; 23:127-36.

14. Ramsay SE, Whincup PH, Watt RG, Tsakos G, Papacosta AO, Lennon LT, et al. Burden of poor oral health in older age: findings from a population-based study of older British men. BMJ Open 2015; 5:e009476.

15. Molarius A, Engstrom S, Flink H, Simonsson B, Tegelberg A. Socioeconomic differences in self-rated oral health and dental care utilisation after the dental care reform in 2008 in Sweden. BMC Oral Health 2014; 14:134-42.

16. Mejia G, Armfield JM, Jamieson LM. Self-rated oral health and oral health-related factors: the role of social inequality. Aust Dent J 2014; 59:226-33

17. Sanders AE, Slade GD. Deficits in perceptions of oral health relative to general health in populations. J Public Health Dent 2006; 66:25562.

18. Matos DL, Lima-Costa MF. Auto-avaliação de saúde bucal entre adultos e idosos residentes na Região Sudeste: resultados do Projeto SB-Brasil, 2003. Cad Saúde Pública 2006; 22:1699-707.

19. Silva SRC, Fernandes RAC. Autopercepção das condições de saúde bucal por idosos. Rev Saúde Pública 2001; 35:349-55.

20. Moura C, Gusmão ES, Santillo PMH, Soares RSC, Cimões R. Autoavaliação da saúde bucal e fatores associados entre adultos em áreas de assentamento rural, Estado de Pernambuco, Brasil. Cad Saúde Pública 2014; 30:611-22.

21. Sanders AE, Spencer AJ. Social inequality in perceived oral health among adults in Australia. Aust N Z J Public Health 2004; 28:159-66.

22. Armfield JM, Mejía GC, Jamieson LM. Socioeconomic and psychosocial correlates of oral health. Int Dent J 2013; 63:202-9.

23. Santos JAF. Esquema de classe para abordar a desigualdade de saúde no Brasil. Cadernos do Itaboraí 2013; 1:39-64.

24. Commission on Social Determinants of Health. The social determinants of health: monitoring, research, and training. In: Commission os Social Determinats of Health, editors. Closing the gap in a generation: health equity through action on the social determinants of health. Geneva: World Health Organization; 2008. p.178-91.
25. Tsuboya T, Aida J, Kawachi I, Katase K, Osaka K. Early life-course socioeconomic position, adult work-related factors and oral health disparities: cross-sectional analysis of the JSHINE study. BMJ Open 2014; 4:e005701.

26. Locker D. Self-esteem and socioeconomic disparities in self-perceived oral health. J Public Health Dent 2009; 69:1-8

27. Damascena GN, Szwarcwald CL, Malta DC, Souza Júnior PRB, Vieira MLFP, Pereira CA, et al. O processo de desenvolvimento da Pesquisa Nacional de Saúde no Brasil, 2013. Epidemiol Serv Saúde 2015; 24:197-206.

28. Freitas MPS. Pesquisa Nacional de Saúde: plano amostral. Rio de Janeiro: Instituto Brasileiro de Geografia e Estatística; 2014.

29. Instituto Brasileiro de Geografia e Estatística. Classificação de ocupações para pesquisas domiciliares - COD, principais diferenças entre a COD e a CBO-Domiciliar. In: $8^{\circ}$ Fórum SIPD. https://ww2.ibge.gov.br/home/estatistica/in dicadores/sipd/oitavo_forum/COD.pdf (acessado em 10/Ago/2016).

30. Rose D, O'Reilly K, Martin J. The ESRC review of government social classifications. Popul Trends 1997; 89:49-89.

31. Instituto Brasileiro de Geografia e Estatística. Pesquisa Nacional de Saúde 2013: indicadores de saúde e mercado de trabalho. Rio de Janeiro: Instituto Brasileiro de Geografia e Estatística; 2016.

32. World Health Organization. From surveys to survillance. In: World Health Organization, organizador. Oral health surveys: basic methods. Geneva: World Health Organization; 2013. p. 65-7.

33. Santos JAF. Classe social e desigualdade de saúde no Brasil. Rev Bras Ciênc Soc 2011; 26:27-56.

34. Landis JR, Koch GG. The measurement of observer agreement for categorical data. Biometrics 1977; 33:159-74.

35. Peker K, Bermek G. Oral health: locus of control, health behavior, self-rated oral health and socio-demographic factors in Istanbul adults. Acta Odontol Scand 2011; 69:54-64.

36. Matos DL, Lima-Costa MF. Autoavaliação da saúde bucal entre adultos e idosos residentes na Região Sudeste: resultados do Projeto SB-Brasil, 2003. Cad Saúde Pública 2006; 22:1699-707.

37. Vale EB, Mendes ACG, Moreira RS. Autopercepção da saúde bucal entre adultos na região Nordeste do Brasil. Rev Saúde Pública 2013; 47 Suppl 3:98-108.

38. Gabardo MCL, Moysés ST, Moysés SJ. Autopercepção de saúde bucal conforme o Perfil de Impacto da Saúde Bucal (OHIP) e fatores associados: revisão sistemática. Rev Panam Salud Pública 2013; 33:439-45.

39. Darin-Mattsson A, Fors S, Kareholt I. Different indicators of socioeconomic status and their relative importance as determinants of health in old age. Int J Equity Health 2017; $16: 173-84$. 
40. Krieger N, Williams DR, Moss NE. Measuring social class in US public health research: concepts, methodologies, and guidelines. Annu Rev Public Health 1997; 18:341-78.

41. Barata RB, Ribeiro MCSA, Silva ZP, Antunes JLF. Classe social: conceitos e esquemas operacionais em pesquisa em saúde. Rev Saúde Pública 2013; 47:647-55.

42. Santos JAF. Uma classificação socioeconômica para o Brasil. Rev Bras Ciênc Sociais 2005; 20:27-46.

43. World Health Organization. Handbook on health inequality monitoring: with a special focus on low- and middle-income countries. Geneva: World Health Organization; 2013.

44. Santos JAF. Classe social, território e desigualdade de saúde no Brasil. Saúde Soc 2018; 27:556-72.
45. Santos JAF. Efeitos de classe na desigualdade racial no Brasil. Dados Rev Ciênc Sociais 2005; 48:21-65.

46. Lundegren N, Axtelius B, Hakansson J, Akerman S. Dental treatment need among 20 to 25-year-old Swedes: discrepancy between subjective and objective need. Acta Odontol Scand 2004; 62:91-6.

47. Cohen-Carneiro F, Souza-Santos R, Rebelo MAB. Quality of life related to oral health: contribution from social factors. Ciênc Saúde Colet 2011; 16 Suppl 1:1007-15.

48. Brasil. Decreto no 7.872 , de 26 de dezembro de 2012. Regulamenta a Lei no 12.382 , de 25 de fevereiro de 2011, que dispõe sobre o valor do salário mínimo e a sua política de valorização de longo prazo. Diário Oficial da União 2002; 26 dez. 


\section{Abstract}

The study analyzed self-rated oral health in the Brazilian adult population according to socioeconomic status (region of residence, schooling, income, and social class), exploring the variables with the greatest sensitivity to measure the association. The study sample included 59,758 individuals 18 years or older who participated in the Brazilian National Health Survey in 2013, a population-based household survey. Self-rated oral health (teeth and gums) was analyzed as positive, fair, or negative. Multinomial logistic regression was used to estimate crude and adjusted odds ratios $(O R)$ and respective $95 \%$ confidence intervals $(95 \%$ CI). Percentage of agreement and kappa values were calculated to compare the values obtained with the regression models and the expected values. Self-rated oral health was positive in $67.4 \%$, fair in $26.7 \%$, and negative in $5.9 \%$. After adjustment, the odds of negative self-rated health were significantly higher in individuals with per capita household income up to one minimum wage, or approximately USD 270/month $(O R=$ 4.71; 95\%CI: 2.84-7.83), without complete primary schooling $(\mathrm{OR}=3.28$; 95\% CI: 2.34-4.61), in the social class devoid of assets $(\mathrm{OR}=3.03 ; 95 \% \mathrm{CI}$ : 2.12-4.32) and residents of Northeast Brazil (OR = 1.50; 95\%CI: 1.19-1.89). Various indicators of socioeconomic status influence self-rated oral health, but per capita household income, schooling, and social class accounted for the largest gradient in self-rated oral health in Brazilian adults in 2013.

Self-Assessment; Oral Health; Health Status Disparities; Social Conditions; Health Surveys

\section{Resumen}

Se analizó la autoevaluación de salud bucal de la población adulta brasileña según la posición socioeconómica (región de residencia, escolaridad, renta y clase social), explorando las variables con mayor sensibilidad para medir tal asociación. Se han estudiado 59.758 individuos con 18 años o más de edad, que participaron en la Encuesta Nacional de Salud 2013, una encuesta domiciliar de base poblacional. La autoevaluación de la salud bucal (dientes y encías) fue analizada como positiva, regular y negativa. Através de la regresión logística multinomial, se estimaron odds ratio (OR) brutos y ajustados y los respectivos intervalos con 95\% de confianza (IC95\%). Se calcularon los porcentajes de concordancia y el valor de kappa para comparar los valores obtenidos por los modelos de regresión y los valores esperados. La prevalencia de la autoevaluación de salud bucal positiva fue 67,4\%, 26,7\% para regular y 5,9\% para negativa. Después del ajuste, la posibilidad de autoevaluar la salud bucal como negativa fue significativamente más elevada entre los individuos con ingresos domiciliarios per cápita de hasta un salario mínimo (OR = 4,71, IC95\%: 2,84-7,83), sin niveles de escolaridad completa $(\mathrm{OR}=3,28$; IC95\%: 2,34-4,61), de la clase social desposeídos de activos $(\mathrm{OR}=3,03$; IC95\%: 2,12-4,32) y residentes de la región Nordeste (OR = 1,50; IC95\%: 1,19-1,89). Diversos indicadores de posición socioeconómica influencian la percepción sobre la salud bucal, pero la renta domiciliar per cápita, la escolaridad $y$ la clase social fueron los responsables del mayor gradiente en la autoevaluación de salud bucal de adultos en Brasil en 2013.

Autoevaluación; Salud Bucal; Disparidades em el Estado de Salud; Condiciones Sociales; Encuestas Epidemiológicas
Recebido em 26/Mai/2018

Versão final reapresentado em 28/Nov/2018

Aprovadoe em 06/Fev/2019 